\title{
Battery, Ultra-capacitor based Hybrid Energy Storage System (HESS) for EV applications with PI and Fuzzy logic controllers
}

\author{
Jonnalagadda Santhosh ${ }^{1}$, Anil Kumar Rajagiri ${ }^{1}$, Prashanth Morri ${ }^{1}$ \\ ${ }^{1}$ Department of EEE, GRIET, Hyderabad
}

\begin{abstract}
The extensive use of vehicles based on IC engines has contributed to serious effects on the environment and accelerated depletion of fossil fuel reserves which has led to a rapid increment in fuel cost over the past few decades. By consideration of these undesirable effects, the automotive industry will move towards the electrified drivetrains for obtaining zero-emission and higher efficiency. In electrified vehicles, the drive is coupled entirely partially by e-motors powered by energy storage sources. The choice of the simple structured HESS topology is to make a better understanding of power flow and controlling circuits based on limits of existing energy storage sources and contribute to the electrification of the drive. The main intention of this paper, the excessive current which is required by the motor is to draw from the ultra-capacitor without burdening of battery. This will improve the efficiency of the storage sources and also smooth running of the drive. This document examines the feasibility and capacity of a HESS which integrates the battery and ultra-capacitor units and controlling through PI and Fuzzy controllers.
\end{abstract}

\section{Introduction}

The electrification of vehicular drives will make results in demanding of better performance and efficiency of Energy Storage System (ESS) for Electric Vehicle (EV) and Hybrid-Electric Vehicle (HEV) drives. Right now, no existence of only one type of energy storage source, which can provide all the requirements of vehicles while moving on roads. Li-ion batteries are a promising new scientific advance for the applications of vehicles because of their large specific-energy and comparatively larger specific-power than batteries of lead-acid and $\mathrm{Ni}$ $\mathrm{MH}$. If the Li-ion battery is subjected to quickly fluctuating charging/discharging currents due to coping with rapid fluctuations in torque and load power, the battery's life can be severely reduced. Ultra-Capacitor (UC) has superior in specific power and lower range of specific energy. By consideration of these properties, we can say Lithium-ion batteries are complement to UltraCapacitors, it is worth combining these energies to give appropriate results to run a drive [1]-[4].

\section{HESS configuration}

The literature on HESS topology has been discussed in [5], [6]. In all the simplest combinations of batteries and supercapacitors, the direct connection of the two units is achieved through a DC bus. Using DC-DC converters and controllers, this topology can be implemented very easily and at a low cost, but UC can't be used to its full potentials in this configuration since voltage of UC unit is restricted by the voltage of battery unit (BU).

The HESS topology is provided with a partiallydecoupled technology with a single DC-dc converter that connects $\mathrm{BU}$ and $\mathrm{UC}$. When especially in comparison towards the scenario of BU as well as UC are connected to the DC link via individual DC-dc converters, this arrangement will result in reduced weight. In this topology the DC link is coupled to either $\mathrm{BU}$ or UC. By considering the topology which the direct connection of BU with DC link and UC and DC bus are interfacing with DC-dc converter, here the unit of UC will be functioning of broader limits of voltage due to the converter and $\mathrm{BU}$ will affects from the rapid change of discharging/charging currents which are required or obtained from the electrified drive during acceleration or regenerative braking conditions respectively. To avoid this affects here we recommend partiallydecoupled HESS design where fig. 1 shows.

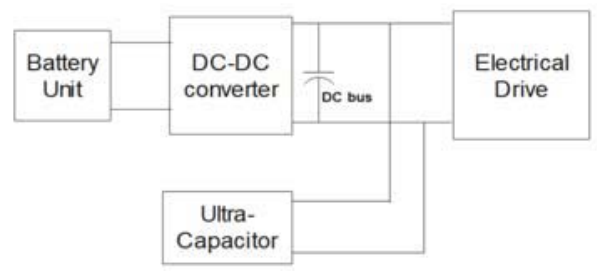

Fig.1. Proposed HESS configuration with Batter Unit, DC-DC Converter, Ultra-Capacitor directly interfaced to DC link. As a result of this study, BU is protected in this configuration by providing the proper control technique to the DC-dc converter. In the contrast to the case where

* Jonnalagadda Santhosh: jsanthosh00@gmail.com 
$\mathrm{BU}$ is interfaces immediate to the $\mathrm{DC}$ link, $\mathrm{UC}$ is kept behind to the converter, a smaller size converter will be used because in fully-decoupled design, the individual converters are required to interfaces $\mathrm{BU}$ as well $\mathrm{UC}$ units with DC link. Here the voltage of DC link is unaffected to the fluctuations, this scheme is economically unattractive due to the use of two DC-dc converters.

In this design we are using DC-dc converter connects between $\mathrm{BU}$ and DC link is a bi-directional means power can be flows in both the directions. Through this converter the battery is able to supply energy during propulsion of drive and absorbs the energy from the regenerative braking.

The simplicity of the bidirectional buck-bust converter makes it most suitable for DC-DC converters with BU and UC interfaces because in addition to performance weight volume and cost must first be considered. BU is linked to the converter's low-potential side and UC is linked to the converter's high-potential side. Due to this alliance of BU on the low-potential side reduces its level of difficulty, addition to that the price of equalizing of cells. By handling the high currents by the converter while using in vehicular application the losses will be more. To reduce this loss, we are using this dual-module interleaved structure of a Bi-directional converter (BDC) where fig. 2 shows. By using this converter, the losses will be reduced by $50 \%$ and also cancellation of ripples in currents. The phase shift between the PWM signals from each DC-dc converter can cause this effect. The double frequency of effective switching compared to normal switching frequency a smaller filter will be used to get less ripple of current at the terminals of BU.

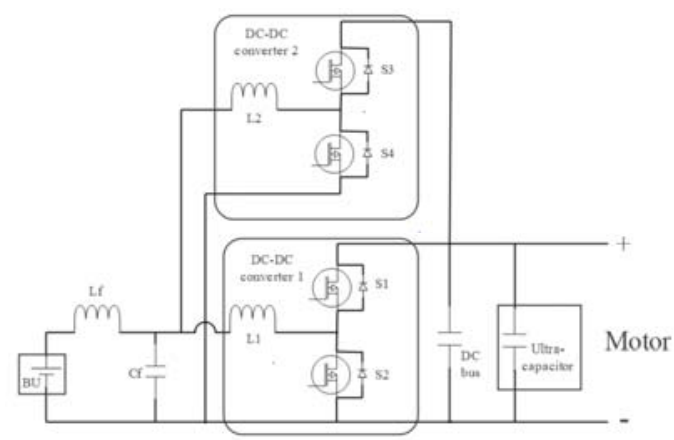

Fig.2. Circuit representation of 2-module, interleaved connection of BDC

Here, $\mathrm{L}_{\mathrm{f}}, \mathrm{C}_{\mathrm{f}}$ are the Inductance and Capacitance of filer components to reduce the ripples of battery.

$\mathrm{S} 1$ and $\mathrm{S} 3$ are the buck switches which are acting during the regenerative braking. S2 and S4 are the boost switches which are acting during propulsion of motor i.e., running conditions of drive.

When the motor needs to rotate, it requires more current, then the Ultra-Capacitor will provide the excess power required by the motor. Under normal conditions only the Battery discharging current meets the requirement of the motor. At this time, the switches S2 and S4 will operate by providing pulses. Therefore, according to the structure of proposed converter Boost (Step-Up) operation is performed by storing the charge in Inductor (L1 and L2) [7].

The output of Boost converter $V_{\text {out }}=V_{\text {in }} /(1-D)$ (1)

The Voltage across the Inductor is $V_{L}=V_{\text {in }}-V_{\text {out }}$ (2)

When braking is applied the current flows from load to source. The Battery and Ultra-Capacitor are ready to charge by taking the regenerative current. In this regeneration process switches S1 and S3 will operate. Here Buck (Step-down) operation is performed.

The output of Buck converter $V_{L}=V_{\text {in }}(D)$

Here $D=T_{\mathrm{ON}} / T$

(Where $\mathrm{D}$ is the Duty ratio).

\section{Controller design}

\subsection{PI controller:}

The BU and UC function is correlated because of HESS energy sharing algorithm. Primarily four considerations are to be taken while preparing of energy sharing algorithm:

(1) In the results shown in fig.13., fig.14., the maximum rate of discharging nature of battery unit current indirectly represents the degradation of battery life [8].

(2) The rate at which the power of the Battery Unit performs at the time intervals of charging/discharging; This value refers at which the rate of change in power of batter $d p / d t$. To protect the battery from the large rate of change of currents at charge/discharge time intervals we should limit the value of $d p / d t$.

(3) For charging of a battery there is mainly two scenarios (i) Maximum charging current for battery and (ii) Maximum charging voltage for voltage: If the grid is providing charging for $\mathrm{BU}$ of the vehicle proportional to the charging scheme of the Constant-Current ConstantVoltage (CCCV). If at the time regenerating braking, the BU get charged in proportional to second scenario which refers to recycling of energy.

(4) Voltage regulation of DC link: The variations of DC link should remain within the range of prescribed limits of input voltage of motors. The main reasons for the malfunctioning of EM system are under-voltage and overvoltage. Hence, BU is controlled to absorb energy from UC or use to charge UC where the requirement of DC link will be maintaining to within prescribed limits. As Fig. 3 represents the PI controller block diagram for the BDC. Here the PI controller is used to control the currents in the Inductors and the DC bus voltage [9], [10]. 


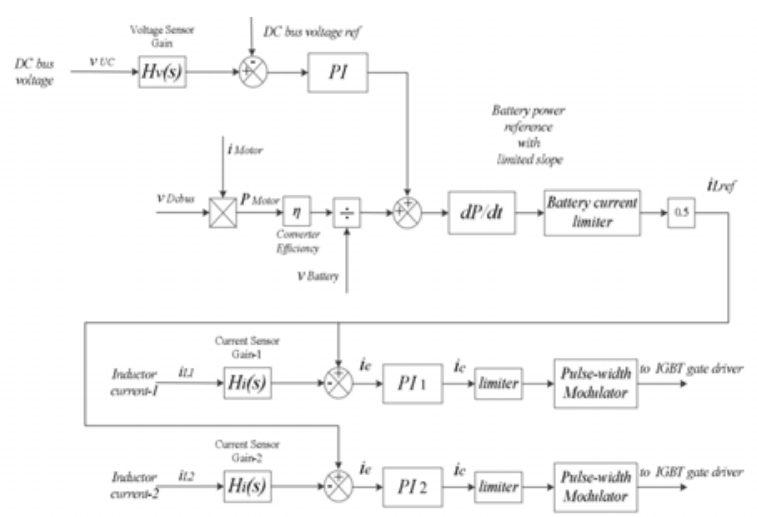

Fig.3. PI controller block diagram representation of converter

The block diagram representation of PI controller for converter controlling is represented in fig.3. Here, the converter controlling is done by controlling the currentscarried by the inductors based on the mode of operation. Here, the individual inductors are regulated by the closely loop arrangement of control system which is shown in Fig.4.

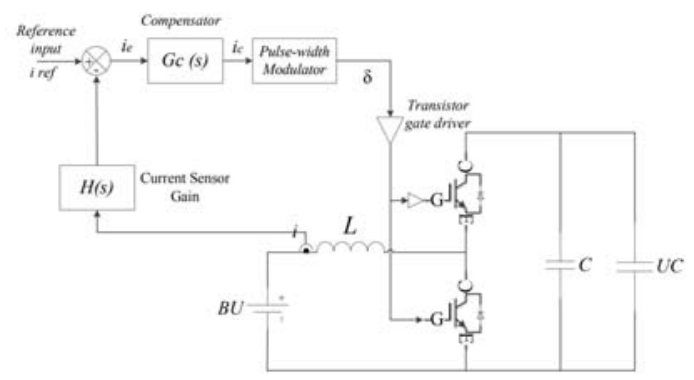

Fig.4. Controlling of current for individual inductors

\subsection{Power calculations:}

According to the vehicle speeds variation between two specific consecutive intervals of time, it can be seen whether the vehicle is accelerating or decelerating. The speed, acceleration/deceleration, and other parameters like rolling resistance, efficiency of the transmission system, mass, air resistance and gear ratio, can be used to calculate the power consumption of the vehicle [11]. The road gradient also taken into consideration when the vehicle is driving. To obtain the required performance the following equations are expressed below:

$$
\begin{aligned}
& \text { Power }=\text { Torque }^{*} w \\
& \text { Torque }^{*} \text { Force }_{e q} * r \\
& \text { Force }_{e q}=F_{a d}+F_{a c}+F_{r r}+F_{g} \\
& F_{a d}=0.5 * \rho v^{2} C_{a d} A \\
& F_{a c}=m a \\
& F_{r r}=C_{r} m g \cos \theta \\
& F_{g}=m g * \sin \theta \\
& w=\frac{v}{r}=\frac{n}{\operatorname{cor}^{2} 60} * 2 \pi
\end{aligned}
$$

The representation of Equation (4) is demand of Power $(\mathrm{P})$ in watts to the Torque obtained by wheels in $\mathrm{Nm}$ and the angular speed $(W)$.

Equation (5) gives the Toque equation of wheels in relation with force equivalent $\left(\right.$ Force $\left._{e q}\right)$, the radius of the wheels in meters.

Equation (6) represents the equivalent force $\left(\right.$ Force $\left._{e q}\right)$, which is addition of forces like rolling resistance, acceleration, aerodynamic drag and gravity forces that will explained in (7) - (10) respectively.

Here, $C_{a d}$ represents the coefficient of drag by body shape of vehicle, $\mathrm{A}\left(\mathrm{m}^{2}\right)$ represents frontal area, $v$ is vehicle's velocity and $\rho$ represents density of air.

In equation (8) - (10) $m(\mathrm{~kg})$ is vehicle mass and $\theta$ the angle between the horizontal plane and surface of road.

The equation (11) represents the angular speed of wheel from the velocity of vehicle $(v)$ and the radius of tire $r$, and also from speed of motor (n) in rpm and gear ratio $C_{\mathrm{g} r}$

Here Fig.5. shows a blocks representation of the energy sharing algorithm used in the controlling of HESS, which contain the conditions which shown in Section 3. At the time of system starts, the controller is always used to monitor the values of the $\mathrm{V}_{\mathrm{BU}}$, the $\mathrm{V}_{\mathrm{UC}}$, the current -carrying by both the inductors in the proposed DC-DC converter and the amount of current which carried to the load from HESS. The values of variables are subject to their respective restrictions based on the limits. When the $\mathrm{V}_{\mathrm{UC}}$ exceeds $45 \mathrm{~V}$, the $\mathrm{UC}$ stored energy will charge the battery by sharing its energy. On the other hand, if the $\mathrm{V}_{\mathrm{UC}}<35 \mathrm{~V}$, then the battery charges the Ultra-capacitor and restores the DC bus voltage. This phenomenon protects the Li-ion battery due to the rapid power Variations while charging/discharging conditions [12] - [14].

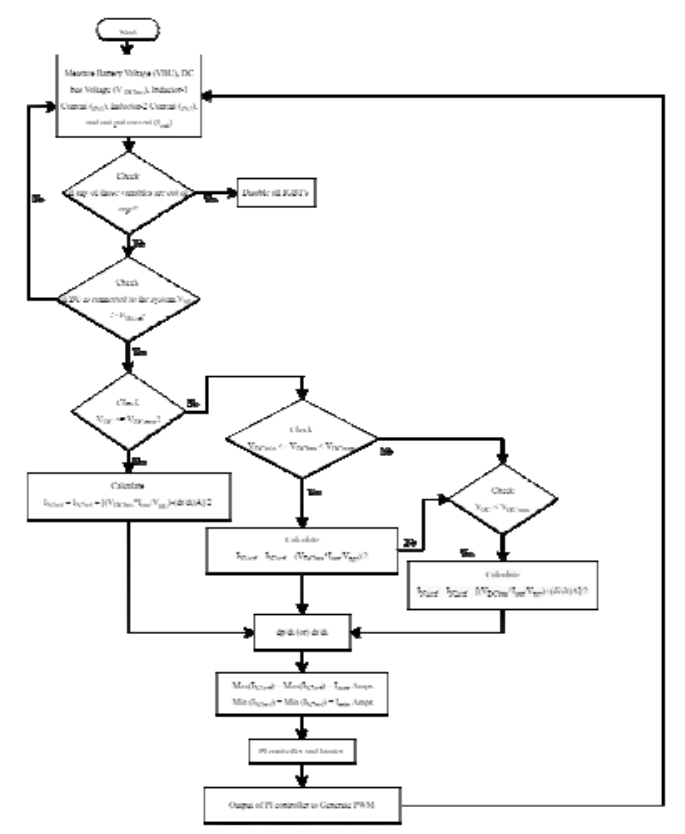

Fig.5. Flow diagram of HESS power management algorithm 


\subsection{Fuzzy Logic Controller:}

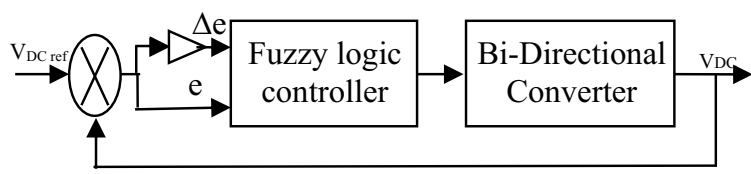

Fig.6. Block diagram representation of Fuzzy controller for BDC

As shown in fig. 6 it represents the block diagram of the fuzzy controller for Bidirectional converter. Fuzzy logic is a mathematical logic based on "degree of truth" unlike the "true or false ". It handles with imprecise or uncertain data. Fuzzy logic controller is easy develop and design and can operate at wider operating conditions. Fuzzy logic is utilized in different industrial automation and household applications.

(If-then statements)

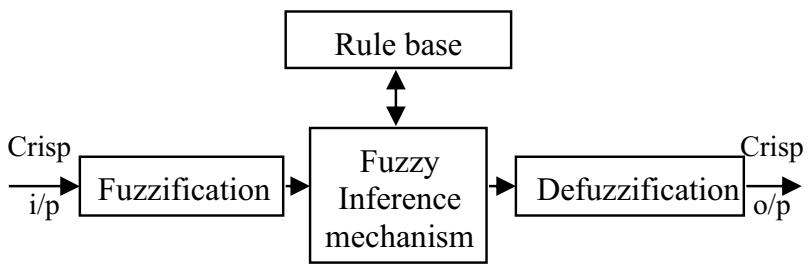

Fig.7.Blocks representation of Fuzzy Controller

Input: values of input of fuzzy controller are nothing but measured output values of system/plant.

Fuzzification: Mapping of membership values between 0 and 1 depends on the value of each date of input nothing but change in error $(\Delta \mathrm{e})$ and value of errors(e).

Rule base: It represents as a combined rules of different types which are used in algorithm of fuzzy controller which are general statements like "if and then". Where the if refers the condition and then refers conclusion.

- The minimization of duty cycle should be done, when $\mathrm{V}_{\mathrm{DC}}>\mathrm{V}_{\text {required }}$

- The increment of duty cycle should be done, when $\mathrm{V}_{\mathrm{DC}}<\mathrm{V}$ required

Here, $V_{D C}$ represents the DC link voltage of converter.

Inference mechanism: Here the different decisions have to be taken to develop a signal that will be sent to plant in order to make it control.

Defuzzification: It is the compliment process of functions carried in fuzzification. A single crisp value of output will be generated by aggregating the all decisions, and by the help of PWM generator the generated crisp value output will be given to the converter. [15] - [17]

To reduce the calculations mostly triangular and trapezoidal based membership functions are used. The membership functions of error and change in error and output are shown in Fig.8, Fig.9 and Fig.10 respectively.

Table 1: Rule base of converter using fuzzy based controller

\begin{tabular}{|c|c|c|c|c|c|c|c|}
\hline e & VS & S & RS & M & RB & B & VB \\
\hline VS & RM & RS & RS & S & S & VS & VS \\
\hline S & RM & RM & RS & RS & S & S & VS \\
\hline RS & M & M & RM & RS & RS & S & S \\
\hline M & RM & VM & M & M & M & RM & RS \\
\hline RB & RB & RB & VM & M & M & RM & RM \\
\hline B & B & B & RB & RB & VM & VM & M \\
\hline VB & VB & B & B & RB & RB & VM & VM \\
\hline
\end{tabular}

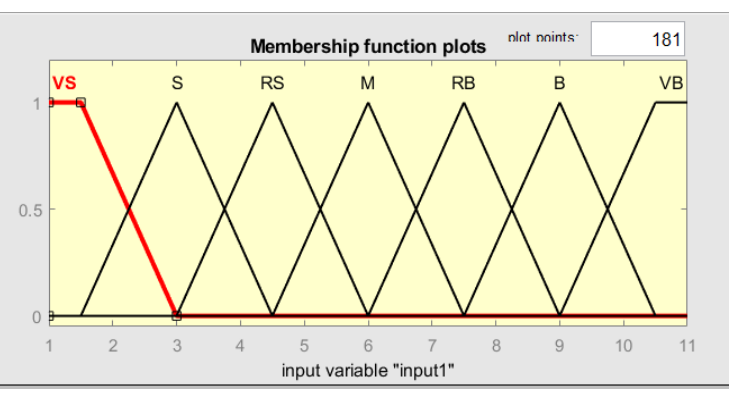

Fig.8. Membership function of input error.

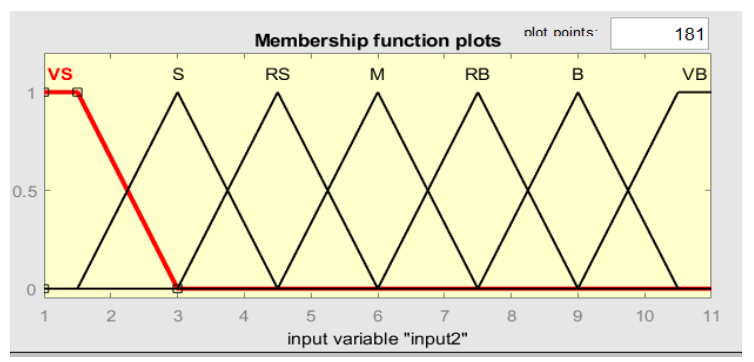

Fig.9. Membership function of change in error.

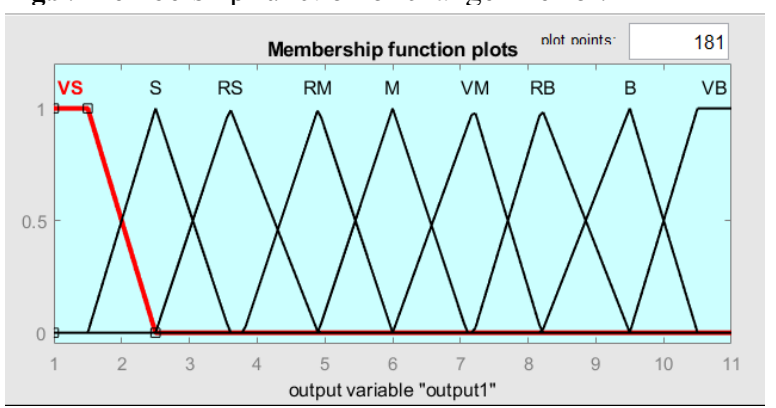

Fig.10. Membership function of change in output. 


\section{Simulation results}

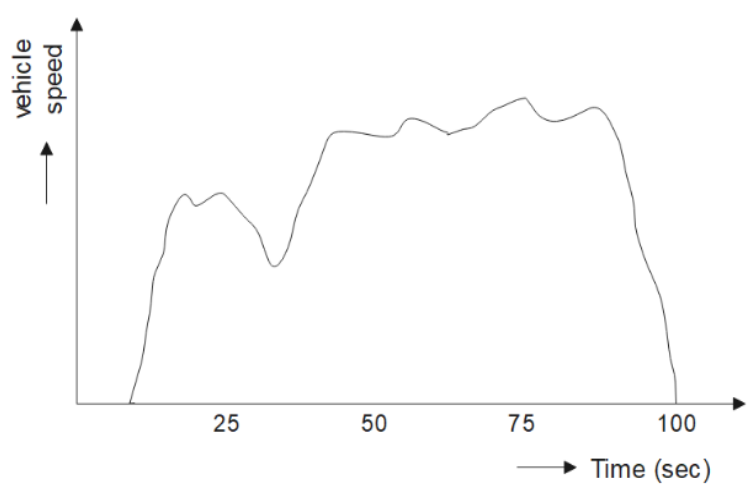

Fig.11. Drive cycle which is used to study up to $100 \mathrm{sec}$ The given drive cycle will be given to the motor to run in proportional to the respective drive cycle.

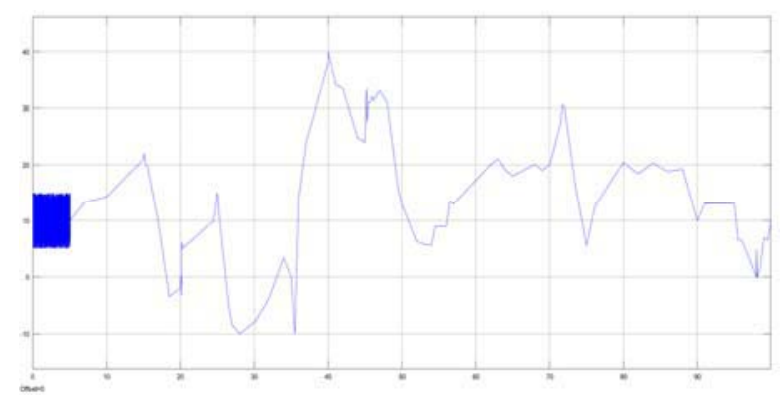

Fig.12. Required current of the motor to run accordingly with the drive cycle.

The absorption of power by the vehicle will be shown in fig. 12. The requirement of power to run a motor will be based on the drive cycle. According to fig.11, Whenever the motor starts rotating it requires high current as per the requirement of high torque. By this, the motor will take more current to start. At the time of braking of motor, the requirement of power of less as shown.

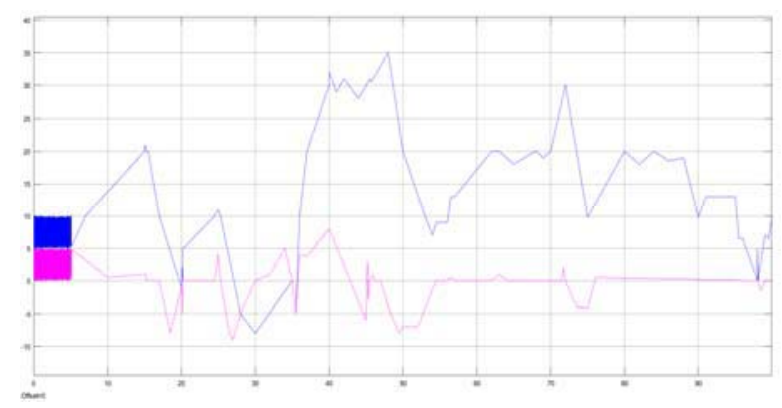

Fig.13. Simulation results of current discharging of BatteryUnit (blue) and Ultra-Capacitor discharging (Pink) using PI Controller.

When the requirement of power is within the limits of $\mathrm{BU}$ power, then the $\mathrm{BU}$ will be the only source to satisfy the rotor running condition. Whenever the $\mathrm{P}_{\text {load }}>$ $\mathrm{P}_{\mathrm{BU}}$ then the UC will be source to provide the power of $\left(\mathrm{P}_{\text {load }}-\mathrm{P}_{\mathrm{BU}}\right)$.

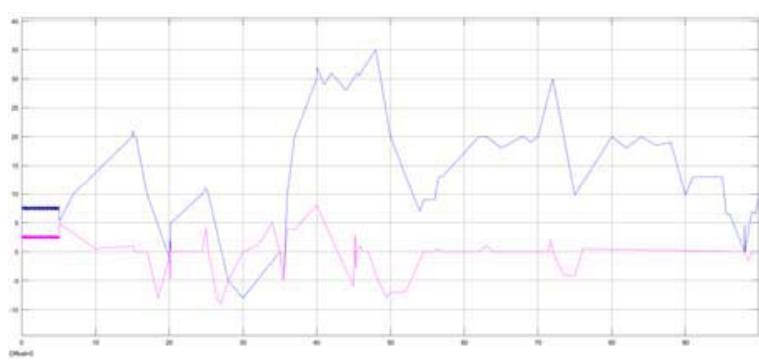

Fig.14. Simulation results of current discharging of BatteryUnit (blue) and Ultra-Capacitor discharging (Pink) using Fuzzy logic controller.

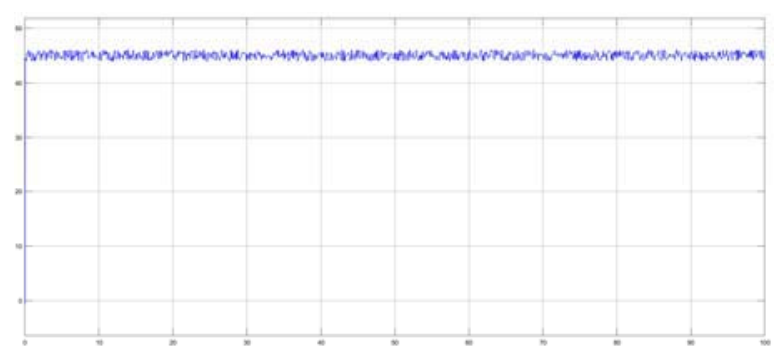

Fig.15. Simulation result of DC link voltage.

Here the simulation results of $\mathrm{V}_{\mathrm{DC}}$ link which is maintains constant because to provide constant voltage to the drive is shown in fig. 15. The algorithm belongs to energy management is mainly used to limit the $\mathrm{V}_{\mathrm{DC}}$ link and discharging of Battery Unit current within the prescribed limits and the limits will be independent on the requirement of power by the load. If the required power of the load is more than the battery discharging limits then the Ultra-Capacitor unit will provide the remaining power where the difference between load requirement and the maximum limit of battery. By the algorithm which is used is not only the Battery discharging Current but also the rate of change of power of the battery unit can be limited by the value of $d p / d t$, above this limit Ultra-Capacitor will be responsible to meet the fast-changing power requirements.

\section{Conclusion}

In this paper, the analysis of PI Controller and Fuzzy based controller for Bi-directional converter to sharing of load current between Battery Unit and UltraCapacitor Unit is studied and implemented in MATLAB/Simulink. The major objective of this research is to understand the Electric vehicle operation and the sources used in EV's like batteries \& UltraCapacitor, properties, and different control methods for Bi-directional converter operation. The simulations results prove both models give the desired output but the model developed using the fuzzy logic controller approach performed slightly better compared to the PI controller in terms of less steady-state. These two controllers are effectively handling the sharing of current between both sources but by considering the factors like error, settling time Fuzzy logic controller is more efficient compare with the PI controller. 


\section{References}

[1] S.M. Lukic, J. Cao, R. C. Bansal, F. Rodriguez, and A. Emadi, IEEE Trans. Ind. Elec., 55, pp. 22582267 (2008).

[2] J. Cao, A. Emadi, IEEE Trans. Pow. Elec., 27,pp. 122-132 (2012).

[3] M. A. Hannan, M. M. Hoque, A. Mohamed and A. Ayod, Ren. and Sust. Engy Rev., 69, pp. 771-789 (2017).

[4] J. Bauman, M. Kazerani, IEEE Tran. Veh. Tech., 57, pp. 760-769, (2008).

[5] A. Ostadi, M. Kazerani, and S.-K. Chen, IEEE Transp. Electrif. Conf. Expo, pp. 1-7 (2013).

[6] J. Sutaria and C. Chauhan, EESCO, pp. 1-6., (2015).

[7] Power Electronics: Circuits, Devices and Applications by M. H. Rashid, Pearson/Prentice Hall, (2004).

[8] G. Ning, B. Haran, and B. N. Popov, J Power Sources. 117, pp. 160-169, May (2003).

[9] J. W. Dixon, M. Ortuzar, and E. Wiechmann, Proc. EVS18, p. 148, (2014).

[10] M. B. Camara, H. Gualous, F. Gustin, and A. Berthon, IEEE Trans. Veh. Tech., 57, pp. 27212735, (2008).

[11] K. Zhuge, M. Kazerani, ITEC pp 1-5, (2014).

[12] N Jinrui, S Fengchun, R Qinglian, IEEE VPPC (2006).

[13] P Aruna, Prabhu V Vasan, ICPEDC, (2019).

[14] J. Cao, N. Schofield, and A. Emadi, IEEE VPPC pp. 1-6, (2008).

[15] R. Nagaraj, ICPE, (2006)

[16] Chetan P Ugale, ISCO, (2017).

[17] Anil Kumar Rajagiri, Sandhya Rani MN, Syed Sarfaraz Nawaz and Suresh Kumar T, SEFET (2019). 